\title{
A Dynamical Behavior of COVID-19 Virus Model with Carrier Effect to Outbreak Epidemic
}

Hassan Fadhil AL-Husseiny

University of Baghdad, College of Science

Ahmed A. Mohsen ( $\nabla$ aamuhseen@gmail.com )

University of Baghdad, College of Education for Pure Science (Ibn Al-Haitham) https://orcid.org/00000003-3812-8918

\section{Xueyong Zhou}

Xinyang Normal University

Keywords: Coronavirus, Covid-19, Stability, Awareness, Media programs, Carrier of disease.

Posted Date: April 23rd, 2020

DOI: https://doi.org/10.21203/rs.3.rs-24219/v1

License: (a) This work is licensed under a Creative Commons Attribution 4.0 International License. Read Full License

Version of Record: A version of this preprint was published at Ibn AL- Haitham Journal For Pure and Applied Sciences on April 23rd, 2020. See the published version at https://doi.org/10.30526/34.2.2624. 


\title{
A Dynamical Behavior of COVID-19 Virus Model with Carrier Effect to Outbreak Epidemic
}

\author{
Hassan Fadhil AL-Husseiny ${ }^{1}$, Ahmed A. Mohsen ${ }^{2}$ and Xueyong Zhou ${ }^{3}$ \\ ${ }^{1}$ Department of Mathematics, College of Science, University of Baghdad, Iraq. \\ E-mail: mathhassanmath@yahoo.com \\ ${ }^{2}$ Department of Mathematics, College of Education for Pure Science (Ibn Al-Haitham), University of \\ Baghdad, Iraq. E-mail: aamuhseen@gmail.com \\ ${ }^{3}$ School of Mathematics and Statistics, Xinyang Normal University, Xinyang 464000, Henan, P.R. \\ China. E-mail: xueyongzhou@xynu.edu.cn
}

\begin{abstract}
In this paper, COVID-19 virus model with effect the carrier to outbreak of epidemic is investigated. The outbreak of Covid-19 virus, is described by a mathematical model divided the population into four classes. The first class describes the susceptible unaware to disease, the second class the susceptible with has aware to disease by media coverage, the third class the carrier individuals and the last class is infected individuals. The existence, uniqueness and bounded-ness of the solutions of the model are discussed. All possible equilibrium points are determined. The locally asymptotically stable of the model is studied. Suitable Lyapunov functions are used to investigate the globally asymptotical stability of the model. Finally, numerical simulation is carried out to confirm the analytical results and understand the effect of varying the parameters on spread of disease.
\end{abstract}

Keywords: Coronavirus, Covid-19, Stability, Awareness, Media programs, Carrier of disease.

\section{Introduction:}

In $14^{\text {th }}$ century Europe, some 25 million people out of a total population of about 100 million died from the Black Death [1]. In 1817 the first cholera pandemic, also known as the first Asiatic cholera pandemic or Asiatic cholera, began near the city of Calcutta and spread throughout Southeast Asia to the Middle East, eastern Africa and the Mediterranean coast see [2,3]. While cholera had spread across India many times previously, this outbreak went further, it reached as far as China and the Mediterranean Sea before subsiding. Hundreds of thousands of people died as a result of this pandemic, including many British soldiers, which attracted European attention. This was the first of several cholera pandemics to sweep through Asia and Europe during the 19th and 20th centuries. This first pandemic spread over an unprecedented range of territory, affecting almost every country in Asia. In November, 2002, the severe acute respiratory syndrome coronavirus (SARS-CoV2) emerged in China causing global anxiety as the outbreak rapidly spread, and by July, 2003, had resulted in over 8000 cases in 26 countries. The SARS epidemic in 2003 reported 8098 cases with 774 deaths, and was eventually brought under control by July, 2003, in a matter of 8 months [4-7]. In December, 2019, a novel coronavirus, named SARS-CoV-2, emerged in Wuhan, China, and led to a rapidly spreading outbreak of coronavirus disease 2019 (COVID-19). By Jan 30, 2020, COVID-19 was declared a public health emergency of international concern. It caused more than 7,000 deaths out of 177,500 cases in the world see [8-12]. In this work, we proposed and studied the effect of awareness by media coverage on COVID-19 pandemic mathematical model dynamics. Further, the effect of direct contact with carrier individuals at first 14 days on outbreak the Coronavirus in the population. 


\section{The Effect of Carrier to outbreak the COVID-19}

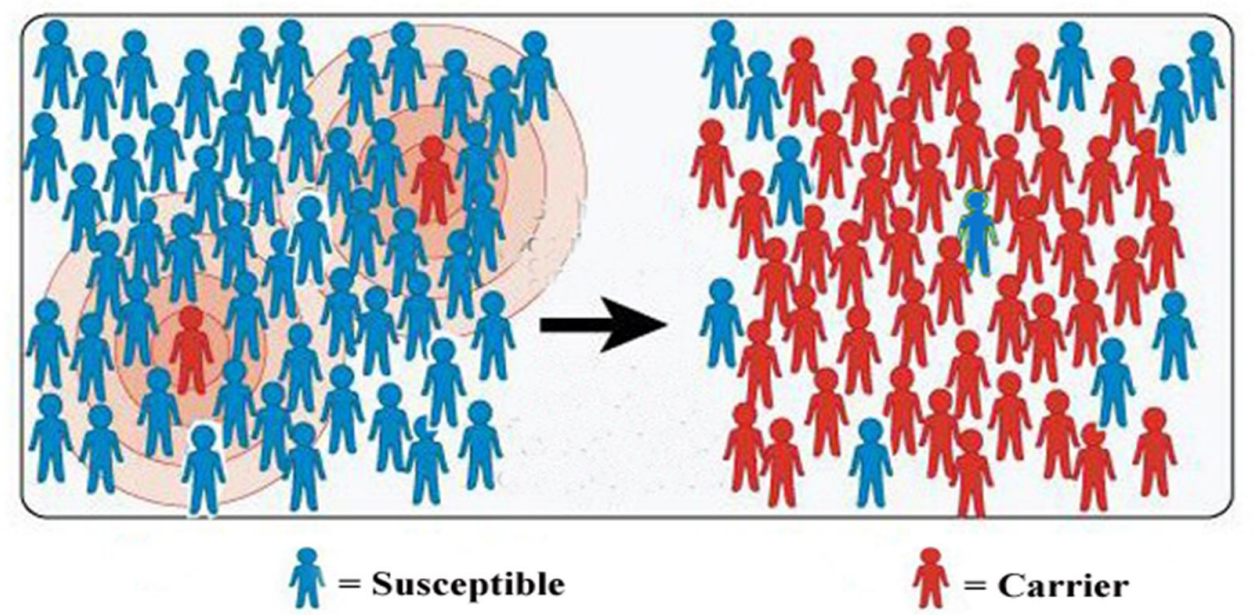

Figure 1: Outbreak Coronavirus due to Carrier by contact

As well, local as well as global stability analysis of the proposed model is also investigated. Finally, we want know the effect of all the parameters as (awareness rate, incidence rate, prevention rate of disease and carrier) by solve this model numerically.

\section{Model Formulation}

We formulate the coronavirus model with effect carrier class on outbreak disease. We can assume that the total population of human denoted by $N$ subdividing into four compartments: unaware susceptible $\left(S_{u}\right)$, aware but still susceptible $\left(S_{a}\right)$, carrier $(C)$, and infected but in quarantine. Thus, $N=S_{u}+S_{a}+C+I$. Let $(v)$ be the coronavirus with grows logistically. We have the following model describes COVID19 virus by nonlinear ordinary differential equations:

$$
\begin{aligned}
& \dot{S_{u}}=\psi-\alpha S_{u}-\beta S_{u} v-\sigma S_{u} C-\mu S_{u} \\
& \dot{S_{a}}=\alpha S_{u}-\beta_{1}(1-\epsilon) S_{a} v-\sigma_{1}(1-\epsilon) S_{a} C-\mu S_{a} \\
& \dot{C}=\beta S_{u} v+\beta_{1}(1-\epsilon) S_{a} v+\sigma S_{u} C+\sigma_{1}(1-\epsilon) S_{a} C-(\mu+\theta+\gamma) C \\
& \dot{I}=\gamma C-(\mu+\theta) I \\
& \dot{v}=r v\left(1-\frac{v}{k}\right)-\mu v
\end{aligned}
$$

Subject to initial conditions $S_{u}>0, S_{a} \geq 0, C \geq 0, I \geq 0, v \geq 0$. in system (1) the birth rate of humans is given by $\Psi>0$. The awareness rate is given by $\alpha \geq 0$. The parameters $\beta \geq 0, \beta_{1} \geq 0, \sigma>0$ and $\sigma_{1}>0$ respectively measure the contacts rate between susceptible with carrier and covid-19 virus. The natural death rate in humans and covid-19 virus is by $\mu>0 . \theta>0$, is the death due to the disease. After (14-20) days, symptoms of the disease begin to appear on the carrier is given by a rate $\gamma>0$. Prevention of disease is given by a rate $(0 \leq \epsilon \leq 1) . r$ and $k$ are respectively, the intrinsic growth rate and carrying capacity of COVID-19 virus.

Theorem (1): All solutions of system (1) which initiate in $R_{+}^{5}$ are uniformly bounded.

Proof: Let $w(T)=(N(t), v(t))$

where $\mathrm{N}(\mathrm{t})=\mathrm{S}_{\mathrm{u}}(\mathrm{t})+S_{a}(\mathrm{t})+\mathrm{C}(\mathrm{t}),+\mathrm{I}(\mathrm{t})$; 
Then

$\frac{d W}{d t}=\left(\frac{d N}{d t}, \frac{d v}{d t}\right)=\left(\psi-\mu \mathrm{S}_{\mathrm{u}}-\mu S_{a}-(\mu+\theta) C-(\mu+\theta) I, r v\left(1-\frac{v}{K}\right)-\mu v\right)$

We know that

$$
\frac{d N}{d t} \leq \psi-q N, \text { where } q=\min \{\mu, \mu+\theta\}
$$

Which implies that

$$
\lim _{t \rightarrow \infty} \sup N(t) \leq \frac{\psi}{q}
$$

While, the last equation of system (1) it follows that

$$
\sup \left(r v\left(1-\frac{v}{k}\right)\right) \leq \frac{r k}{4}
$$

Hence

$$
\frac{d v}{d t} \leq \frac{r k}{4}-\mu v
$$

So that

$$
\lim _{t \rightarrow \infty} \sup v(t) \leq \frac{r k}{4 \mu}
$$

This completes the proof of theorem

\section{Existence of equilibrium points}

It is easy that the infected population $I$ is related with carrier population only. Hence for fixed value of $C$, the value of $I$ can be determined directly by solving the fourth equation in system (1). Then, we can calculate the value of $I$ the following form

$$
I=\frac{\gamma C_{*}}{\mu+\theta}
$$

Consequently, for simplifying, system (1) can be reduced to the following system, in which we can determine the value of $I$, by solving it,

$$
\begin{aligned}
& \dot{S_{u}}=\psi-\alpha S_{u}-\beta S_{u} v-\sigma S_{u} C-\mu S_{u} \\
& \dot{S_{a}}=\alpha S_{u}-\beta_{1}(1-\epsilon) S_{a} v-\sigma_{1}(1-\epsilon) S_{a} C-\mu S_{a} \\
& \dot{C}=\beta S_{u} v+\beta_{1}(1-\epsilon) S_{a} v+\sigma S_{u} C+\sigma_{1}(1-\epsilon) S_{a} C-(\mu+\theta+\gamma) C \\
& \dot{v}=\operatorname{rv}\left(1-\frac{v}{k}\right)-\mu v
\end{aligned}
$$

Now, we can calculate the reproduction number of system (3) and denoted by $\mathcal{R}_{\text {。 }}$, such that

$$
\mathcal{R}_{\circ}=\frac{\sigma S_{u 0}}{\mu+\theta+\gamma}+\frac{r}{\mu}
$$

Therefore, system (3) has at most three biologically feasible points, namely, $E_{i}=$ $\left(S_{u i}, S_{a i}, C_{i}, v_{i}\right), i=0,1,2$. The existence conditions for each of these equilibrium points are discussed in following:

- In the absence of COVID-19 virus, that is $S_{a}=C=v=0$. Then, system (3) has a unique positive equilibrium point, namely COVID-19 free equilibrium point, which denoted by $E_{0}=\left(S_{u 0}, 0,0,0\right)$ where

$S_{u 0}=\frac{\Psi}{\mu}$

provided that the following condition holds

$\alpha=0$

- In the absence of carrier $(C=0)$. Hence, system (3) has an equilibrium point namely carrier free equilibrium point, which denoted by $E_{1}=\left(S_{u 1}, S_{a 1}, 0, v_{1}\right)$ where: 


$$
\begin{aligned}
S_{u 1} & =\frac{\psi}{\alpha+\mu} ; \\
S_{a 1} & =\frac{\alpha \psi}{\mu(\alpha+\mu)} ; \\
v_{1} & =\frac{(r-\mu) k}{r}
\end{aligned}
$$

exists under the condition

$$
r>\mu \text { and } \beta=\beta_{1}=0
$$

- The endemic equilibrium point or COVID-19 equilibrium point, which denoted by $E_{2}=\left(S_{u 2}, S_{a 2}, C_{2}, v_{2}\right)$, where

$$
\begin{aligned}
& S_{u 2}=\frac{r \psi}{r \alpha+\beta(r-\mu) k+r \mu+r \sigma C} ; \\
& S_{a 2}=\frac{\alpha r^{2} \psi}{A_{1} C^{2}+A_{2} C+A_{3}} ; \\
& v_{2}=\frac{(r-\mu) k}{r}
\end{aligned}
$$

here

$$
\begin{aligned}
& A_{1}=\sigma_{1}(1-\epsilon) r^{2} \sigma \\
& A_{2}=\beta_{1}(1-\epsilon)(r-\mu) k r \sigma+\sigma_{1}(1-\epsilon) r[r \alpha+\beta(r-\mu) k+r \mu]+r^{2} \mu \sigma \\
& A_{3}=\left[\beta_{1}(1-\epsilon)(r-\mu) k+r \mu\right][r \alpha+\beta(r-\mu) k+r \mu]
\end{aligned}
$$

exists under the condition

$$
r>\mu
$$

Now, substituting the equation (7a), in $3^{\text {rd }}$ equation of system (3), and simplify that result we get

Where

$$
D_{1} C^{4}+D_{2} C^{3}+D_{3} C^{2}+D_{4} C+D_{5}=0
$$

$$
\begin{aligned}
D_{1}= & -(\gamma+\theta+\mu) r^{2} \sigma A_{1}<0 \\
D_{2}= & \sigma r^{2} \psi A_{1}-(\gamma+\theta+\mu)\left(r^{2} \alpha+\beta(r-\mu) k r+r^{2} \mu\right) A_{1}-(\gamma+\theta+\mu) r^{2} \sigma A_{2} \\
D_{3}= & \beta(r-\mu) k r \psi A_{1}+\sigma r^{2} \psi A_{2}+\sigma_{1}(1-\epsilon) \alpha r^{4} \psi \sigma \\
& \quad-(\gamma+\theta+\mu)\left(r^{2} \alpha+\beta(r-\mu) k r+r^{2} \mu\right) A_{2}-(\gamma+\theta+\mu) r^{2} \sigma A_{3} \\
D_{4}= & \beta(r-\mu) k r \psi A_{2}+\beta_{1}(1-\epsilon)(r-\mu) k \alpha r^{3} \psi \sigma+\sigma r^{2} \psi A_{3} \\
& +\sigma_{1}(1-\epsilon) \alpha r^{3} \psi(r \alpha+\beta(r-\mu) k r+\mu r) \\
& -(\gamma+\theta+\mu)\left(r^{2} \alpha+\beta(r-\mu) k r+r^{2} \mu\right) \\
D_{5}= & \beta(r-\mu) k r \psi A_{3}+\beta_{1}(1-\epsilon)(r-\mu) k \alpha r^{2} \psi(r \alpha+\beta(r-\mu) k+r \mu)>0
\end{aligned}
$$

Obviously, the endemic equilibrium point exists unique if and only if the following condition holds

$$
D_{3}>0, D_{4}>0
$$

OR

$$
D_{2}<0, D_{3}<0
$$

\section{Local stability analysis}

In this section, the local stability analysis of the all equilibrium points $E_{i}, i=0,1,2$ of system (3) studied as shown in the following theorems.

Theorem (2): The COVID-19 free equilibrium point $E_{0}$ of the system (3) is locally asymptotically if the following condition is satisfied

$$
\mathcal{R}_{\circ}<1
$$

Proof: The Jacobian matrix of system (3) at $E_{0}$ can be written as 


$$
J\left(S_{u 0}, 0,0,0\right)=\left(\begin{array}{cccc}
-\mu & 0 & -\sigma S_{u 0} & -\beta S_{u 0} \\
0 & -\mu & 0 & 0 \\
0 & 0 & \sigma S_{u 0}-(\gamma+\theta+\mu) & \beta S_{u 0} \\
0 & 0 & 0 & r-\mu
\end{array}\right)
$$

Thus, the eigenvalues of $J\left(E_{0}\right)$ are given by

$$
\begin{aligned}
& \lambda_{1}=\lambda_{2}=-\mu<0 \\
& \lambda_{3}=\sigma S_{u 0}-(\gamma+\theta+\mu) \\
& \lambda_{4}=r-\mu
\end{aligned}
$$

It is easy from above to verify that condition (9) guarantees that the eigenvalue $\lambda_{3}$ and $\lambda_{4}$ are negative. Then, $E_{0}$ is locally asymptotically stable. However, it is saddle point otherwise.

Theorem (3): The carrier free equilibrium point $E_{1}$ of the system (3) is locally asymptotically if the following conditions are satisfied

$$
\begin{aligned}
& \sigma S_{u 1}+\sigma_{1}(1-\epsilon) S_{a 1}<(\gamma+\theta+\mu) \\
& k r<2 r v_{1}+k \mu
\end{aligned}
$$

Proof: The Jacobian matrix of system (3) at $E_{1}$ can be written as $J\left(S_{u 1}, S_{a 1}, 0, v_{1}\right)=$

$$
\left(\begin{array}{cccc}
-(\alpha+\mu) & 0 & -\sigma S_{u 1} & 0 \\
\alpha & -\mu & -\sigma_{1}(1-\epsilon) S_{a 1} & 0 \\
0 & 0 & \sigma S_{u 1}+\sigma_{1}(1-\epsilon) S_{a 1}-(\gamma+\theta+\mu) & 0 \\
0 & 0 & 0 & \frac{-2 r B_{1}}{k}+(r-\mu)
\end{array}\right)
$$

Thus the eigenvalues of $J\left(E_{1}\right)$ are written by

$$
\begin{aligned}
& \lambda_{1}=-(\alpha+\mu)<0 ; \\
& \lambda_{2}=-\mu<0 ; \\
& \lambda_{3}=\sigma S_{u 1}+\sigma_{1}(1-\epsilon) S_{a 1}-(\gamma+\theta+\mu) ; \\
& \lambda_{4}=\frac{-2 r v_{1}}{k}+(r-\mu)
\end{aligned}
$$

It is easy from above to verify that conditions (10a) and (10b) guarantees that the eigenvalues $\lambda_{i}, i=3,4$ are negative. Then, $E_{1}$ is locally asymptotically stable. However, it is saddle point otherwise.

Theorem (4): The COVID-19 equilibrium point $E_{2}$ of the system (3) is locally asymptotically if the following conditions are hold

$$
\begin{aligned}
& 2 \sigma S_{u 2}+2 \sigma_{1}(1-\epsilon) S_{a 2}<\gamma+\theta+\mu \\
& k\left(2 \beta S_{u 2}+2 \beta_{1}(1-\epsilon) S_{a 2}+r\right)<2 r v_{2}+k \mu
\end{aligned}
$$

Proof: The Jacobian matrix of system (3) at $E_{2}$ can be written as

Here

$$
J\left(S_{u 2}, S_{a 2}, C_{2}, v_{2}\right)=\left(\begin{array}{cccc}
b_{11} & 0 & b_{13} & b_{14} \\
b_{21} & b_{22} & b_{23} & b_{24} \\
b_{31} & b_{32} & b_{33} & b_{34} \\
0 & 0 & 0 & b_{44}
\end{array}\right)
$$




$$
\begin{aligned}
& b_{11}=-\left(\alpha+\beta v_{2}+\sigma C_{2}+\mu\right) ; b_{13}=-\sigma S_{u 2} ; b_{14}=-\beta S_{u 2} ; \\
& b_{21}=\alpha ; b_{22}=-\left(\beta_{1}(1-\epsilon) v_{2}+\sigma_{1}(1-\epsilon) C_{2}+\mu\right) ; \\
& b_{23}=-\sigma_{1}(1-\epsilon) S_{a 2} ; b_{24}=-\beta_{1}(1-\epsilon) S_{a 2} \\
& b_{31}=\beta v_{2}+\sigma C_{2} ; b_{32}=\beta_{1}(1-\epsilon) v_{2}+\sigma_{1}(1-\epsilon) C_{2} ; \\
& b_{33}=\sigma S_{u 2}+\sigma_{1}(1-\epsilon) S_{a 2}-(\gamma+\theta+\mu) ; b_{34}=\beta S_{u 2}+\beta_{1}(1-\epsilon) S_{a 2} \\
& b_{44}=\frac{-2 r v_{2}}{k}+(r-\mu) ; b_{12}=b_{41}=b_{42}=b_{43}=0
\end{aligned}
$$

Now, according to Gersgorin theorem [13], if the following condition holds:

$$
\left|b_{i i}\right|>\sum_{\substack{i=1 \\ i \neq j}}^{4}\left|b_{i j}\right|=P_{i}
$$

Then all eigenvalues of $J\left(E_{2}\right)$ exists in the region:

$$
\Omega=U\left\{U^{*} \epsilon C:\left|U^{*}-b_{i i}\right|<\sum_{\substack{i=1 \\ i \neq j}}^{4}\left|b_{i j}\right|\right\}
$$

Then, all the eigenvalues of $J\left(E_{2}\right)$ exists in the disc centered at $b_{i i}$ with radius $P_{i}$. Thus if the diagonal elements are negative and the condition (11a) holds, all the eigenvalues will be exist in the left half plane and the $E_{2}$ of system (3) is locally asymptotically stabile. Clearly conditions (11a)-(11b) guarantee the existence of all eigenvalues in the left half plane and the proof follows.

\section{Global stability analysis}

The propose of this section is investigate the global stability of COVID-19 virus model given by equations in system (3) near COVID-19 free, carrier free and COVID-19 points respectively given by $E_{i}, i=0,1,2$. We obtain the result in the following theorems

Theorem (5): If the $E_{0}$ is locally asymptotically stable, then it is globally asymptotically stable provided that the following conditions hold:

$$
S_{u 0}<\min \left\{\frac{\gamma+\theta+\mu}{\sigma}, \frac{\mu-r}{\beta}\right\}
$$

Proof: Consider the following function

$$
V_{0}\left(S_{u}, S_{a}, C, v\right)=\left(S_{u}-S_{u 0}-S_{u 0} \ln \frac{S_{u}}{S_{u 0}}\right)+S_{a}+C+v
$$

Clearly, $V_{0}: R_{+}^{4} \rightarrow R$ is a continuously differentiable function such that $V_{0}\left(S_{u 0}, 0,0,0\right)=0$ and $V_{0}\left(S_{u}, S_{a}, C, v\right)>0, \forall\left(S_{u}, S_{a}, C, v\right) \neq\left(S_{u 0}, 0,0,0\right)$. Further, we have

$$
\begin{aligned}
\frac{d V_{0}}{d t}= & \left(\frac{S_{u}-S_{u 0}}{S_{u}}\right)\left[\psi-\beta S_{u} v-\sigma S_{u} C-\mu S_{u}\right]+\left[-\beta_{1}(1-\epsilon) S_{a} v-\sigma_{1}(1-\epsilon) S_{a} C-\mu S_{a}\right] \\
& +\left[\beta S_{u} v+\beta_{1}(1-\epsilon) S_{a} v+\sigma S_{u} C+\sigma_{1}(1-\epsilon) S_{a} C-(\gamma+\theta+\mu) C\right] \\
& +\left[(r-\mu) v-\frac{r}{k} v^{2}\right]
\end{aligned}
$$

Now, by doing some algebraic manipulation and using the conditions (9a) and (12), we get

$$
\frac{d V_{0}}{d t} \leq \frac{-\mu}{S_{u}}\left(S_{u}-S_{u 0}\right)^{2}-\mu S_{a}-\left[(\gamma+\theta+\mu)-\sigma S_{u 0}\right] C-\frac{r}{k} v^{2}-\left[(\mu-r)-\beta S_{u 0}\right] v
$$


Obviously, $\dot{V}_{0}=0$ at $E_{0}=\left(S_{u 0}, 0,0,0\right)$, moreover $\dot{V}_{0}<0$ otherwise. Hence $\dot{V}_{0}$ is negative definite and then the solution starting from any initial point satisfy the conditions (9a) and (12), will approaches asymptotically to COVID-19 free equilibrium point. Hence the proof is complete.

Theorem (6): Assume that $E_{1}$ is locally asymptotically stable, then it is globally asymptotically stable provided that the following conditions hold:

$$
\begin{aligned}
& \sigma S_{u 1}+\sigma_{1}(1-\epsilon) S_{a 1}<\gamma+\theta+\mu \\
& k r<r v_{1} v+k \mu \\
& \left(\frac{\alpha}{s_{a}}\right)^{2}<4\left(\frac{\alpha+\mu}{S_{u}}\right)\left(\frac{\mu}{s_{a}}\right)
\end{aligned}
$$

Proof: Consider the following function

$$
\begin{aligned}
V_{1}\left(S_{u}, S_{a}, C, v\right)= & \left(S_{u}-S_{u 1}-S_{u 1} \ln \frac{S_{u}}{S_{u 1}}\right)+\left(S_{a}-S_{a 1}-S_{a 1} \ln \frac{S_{a}}{S_{a 1}}\right)+C \\
& +\left(v-v_{1}-v_{1} \ln \frac{v}{v_{1}}\right)
\end{aligned}
$$

Clearly, $V_{1}: R_{+}^{4} \rightarrow R$ is a continuously differentiable function such that $V_{1}\left(S_{u 1}, S_{a 1}, 0, v_{1}\right)=0$ and $V_{1}\left(S_{u}, S_{a}, C, v\right)>0, \forall\left(S_{u}, S_{a}, C, v\right) \neq\left(S_{u 1}, S_{a 1}, 0, v_{1}\right)$. further, we have

$$
\begin{aligned}
\frac{d V_{1}}{d t}= & \left(\frac{S_{u}-S_{u 1}}{S_{u}}\right)\left[\psi-\alpha S_{u}-\sigma S_{u} C-\mu S_{u}\right]+\left(\frac{S_{a}-S_{a 1}}{S_{a}}\right)\left[\alpha S_{u}-\sigma_{1}(1-\epsilon) S_{a} C-\mu S_{a}\right] \\
& +\left[\sigma S_{u} C+\sigma_{1}(1-\epsilon) S_{a} C-(\gamma+\theta+\mu) C\right]+\left(\frac{v-v_{1}}{v}\right)\left[(r-\mu) v-\frac{r}{k} v^{2}\right]
\end{aligned}
$$

By using the above conditions (13a)-(13c), we obtained that

$$
\begin{aligned}
\frac{d V_{1}}{d t} \leq & -\left[\sqrt{\frac{\alpha+\mu}{S_{u}}}\left(S_{u}-S_{u 1}\right)-\sqrt{\frac{\mu}{S_{a}}}\left(S_{a}-S_{a 1}\right)\right]^{2} \\
& -\left[(\gamma+\theta+\mu)-\left(\sigma S_{u 1}+\sigma_{1}(1-\epsilon) S_{a 1}\right)\right] C-\frac{r}{k} v\left(v-v_{1}\right)^{2} \\
& -\left[\frac{r}{k} v_{1}-\frac{(r-\mu)}{v}\right]\left(v-v_{1}\right)^{2}
\end{aligned}
$$

Clearly, $\dot{V}_{1}=0$ at $E_{1}=\left(S_{u 1}, S_{a 1}, 0, v_{1}\right)$, moreover $\dot{V}_{1}<0$ otherwise. Hence $\dot{V}_{1}$ is negative definite and then the solution starting from any initial point satisfy the conditions (13a)-(13c), will approaches asymptotically to carrier free equilibrium point. Hence the proof is complete.

Theorem (7): Assume that $E_{2}$ is locally asymptotically stable, then it is globally asymptotically stable provided that the following conditions hold:

$$
\begin{aligned}
& \sigma S_{u 2}+\sigma_{1}(1-\epsilon) S_{a 2}<\gamma+\theta+\mu \\
& k(r-\mu)<r\left(v+v_{2}\right) \\
& (\alpha)^{2}<\frac{4}{9}(\alpha+\mu+\beta v+\sigma C)\left(\beta_{1}(1-\epsilon) v+\sigma_{1}(1-\epsilon) C+\mu\right) \\
& \left(\sigma S_{u 2}-\beta v-\sigma C\right)^{2}<\frac{4}{9}(\alpha+\mu+\beta v+\sigma C) \\
& \quad \times\left(\gamma+\theta+\mu-\left(\sigma S_{u 2}+\sigma_{1}(1-\epsilon) S_{a 2}\right)\right)
\end{aligned}
$$




$$
\begin{aligned}
& \left(\beta S_{u 2}\right)^{2}<\frac{4}{9}(\alpha+\mu+\beta v+\sigma C)\left(\frac{r}{k}\left(v+v_{2}\right)-(r-\mu)\right) \\
& \left(\sigma_{1}(1-\epsilon) S_{a 2}-\beta_{1}(1-\epsilon) v-\sigma_{1}(1-\epsilon) C\right)^{2}<\frac{4}{9}\left(\beta_{1}(1-\epsilon) v+\sigma_{1}(1-\epsilon) C+\mu\right) \\
& \times\left(\gamma+\theta+\mu-\left(\sigma S_{u 2}+\sigma_{1}(1-\epsilon) S_{a 2}\right)\right) \\
& \left(\beta_{1}(1-\epsilon) S_{a 2}\right)^{2}<\frac{4}{9}\left(\beta_{1}(1-\epsilon) v+\sigma_{1}(1-\epsilon) C+\mu\right) \\
& \times\left(\frac{r}{k}\left(v+v_{2}\right)-(r-\mu)\right) \\
& \left(\beta S_{u 2}+\beta_{1}(1-\epsilon) S_{a 2}\right)^{2}<\frac{4}{9}\left(\gamma+\theta+\mu-\left(\sigma S_{u 2}+\sigma_{1}(1-\epsilon) S_{a 2}\right)\right) \\
& \times\left(\frac{r}{k}\left(v+v_{2}\right)-(r-\mu)\right)
\end{aligned}
$$

Proof: Consider the following function

$$
V_{2}\left(S_{u}, S_{a}, C, v\right)=\frac{\left(S_{u}-S_{u 2}\right)^{2}}{2}+\frac{\left(S_{a}-S_{a 2}\right)^{2}}{2}+\frac{\left(C-C_{2}\right)^{2}}{2}+\frac{\left(v-v_{2}\right)^{2}}{2}
$$

Clearly, $V_{2}: R_{+}^{4} \rightarrow R$ is a continuously differentiable function such that $V_{2}\left(S_{u 2}, S_{a 2}, C_{2}, v_{2}\right)=0$ and $V_{1}\left(S_{u}, S_{a}, C, v\right)>0, \forall\left(S_{u}, S_{a}, C, v\right) \neq\left(S_{u 2}, S_{a 2}, C_{2}, v_{2}\right)$.

$$
\begin{aligned}
\frac{d V_{2}}{d t}= & \left(S_{u}-S_{u 2}\right)\left[\psi-\alpha S_{u}-\beta S_{u} v-\sigma S_{u} C-\mu S_{u}\right] \\
& +\left(S_{a}-S_{a 2}\right)\left[\alpha S_{u}-\beta_{1}(1-\epsilon) S_{a} v-\sigma_{1}(1-\epsilon) S_{a} C-\mu S_{a}\right] \\
& +\left(C-C_{2}\right)\left[\beta S_{u} v+\beta_{1}(1-\epsilon) S_{a} v+\sigma S_{u} C+\sigma_{1}(1-\epsilon) S_{a} C-(\gamma+\theta+\mu) C\right] \\
& \quad+\left(v-v_{2}\right)\left[(r-\mu) v-\frac{r}{k} v^{2}\right]
\end{aligned}
$$

Furthermore by taking the derivative with respect to the time and simplifying the resulting terms, we get that

$$
\begin{aligned}
\frac{d V_{2}}{d t}=- & {\left[\frac{q_{11}}{3}\left(S_{u}-S_{u 2}\right)^{2}-q_{12}\left(S_{u}-S_{u 2}\right)\left(S_{a}-S_{a 2}\right)+\frac{q_{22}}{3}\left(S_{a}-S_{a 2}\right)^{2}\right] } \\
& -\left[\frac{q_{11}}{3}\left(S_{u}-S_{u 2}\right)^{2}+q_{13}\left(S_{u}-S_{u 2}\right)\left(C-C_{2}\right)+\frac{q_{33}}{3}\left(C-C_{2}\right)^{2}\right] \\
& -\left[\frac{q_{11}}{3}\left(S_{u}-S_{u 2}\right)^{2}+q_{14}\left(S_{u}-S_{u 2}\right)\left(v-v_{2}\right)+\frac{q_{44}}{3}\left(v-v_{2}\right)^{2}\right] \\
& -\left[\frac{q_{22}}{3}\left(S_{a}-S_{a 2}\right)^{2}+q_{23}\left(S_{a}-S_{a 2}\right)\left(C-C_{2}\right)+\frac{q_{33}}{3}\left(C-C_{2}\right)^{2}\right] \\
& -\left[\frac{q_{22}}{3}\left(S_{a}-S_{a 2}\right)^{2}+q_{24}\left(S_{a}-S_{a 2}\right)\left(v-v_{2}\right)+\frac{q_{44}}{3}\left(v-v_{2}\right)^{2}\right] \\
& -\left[\frac{q_{33}}{3}\left(C-C_{2}\right)^{2}-q_{34}\left(C-C_{2}\right)\left(v-v_{2}\right)+\frac{q_{44}}{3}\left(v-v_{2}\right)^{2}\right]
\end{aligned}
$$

Consequently by using the above conditions we get that 
Where

$$
\begin{aligned}
\frac{d V_{2}}{d t} \leq & -\left[\sqrt{\frac{q_{11}}{3}}\left(S_{u}-S_{u 2}\right)-\sqrt{\frac{q_{22}}{3}}\left(S_{a}-S_{a 2}\right)\right]^{2} \\
& -\left[\sqrt{\frac{q_{11}}{3}}\left(S_{u}-S_{u 2}\right)+\sqrt{\frac{q_{33}}{3}}\left(C-C_{2}\right)\right]^{2} \\
& -\left[\sqrt{\frac{q_{11}}{3}}\left(S_{u}-S_{u 2}\right)+\sqrt{\frac{q_{44}}{3}}\left(v-v_{2}\right)\right]^{2} \\
& -\left[\sqrt{\frac{q_{22}}{3}}\left(S_{a}-S_{a 2}\right)+\sqrt{\frac{q_{33}}{3}}\left(C-C_{2}\right)\right]^{2} \\
& -\left[\sqrt{\frac{q_{22}}{3}}\left(S_{a}-S_{a 2}\right)+\sqrt{\frac{q_{44}}{3}}\left(v-v_{2}\right)\right]^{2} \\
& -\left[\sqrt{\frac{q_{33}}{3}}\left(C-C_{2}\right)-\sqrt{\frac{q_{44}}{3}}\left(v-v_{2}\right)\right]^{2}
\end{aligned}
$$

$$
\begin{aligned}
& q_{11}=\alpha+\mu+\beta v+\sigma C, \quad q_{12}=\alpha, \quad q_{22}=\beta_{1}(1-\epsilon) v+\sigma_{1}(1-\epsilon) C+\mu \\
& q_{13}=\sigma S_{u 2}-\beta v-\sigma C, \quad q_{33}=\gamma+\theta+\mu-\left(\sigma S_{u 2}+\sigma_{1}(1-\epsilon) S_{a 2}\right) \\
& q_{14}=\beta S_{u 2}, \quad q_{24}=\beta_{1}(1-\epsilon) S_{a 2}, \quad q_{44}=\frac{r}{k}\left(v+v_{2}\right)-(r-\mu) \\
& q_{23}=\sigma_{1}(1-\epsilon) S_{a 2}-\beta_{1}(1-\epsilon) v-\sigma_{1}(1-\epsilon) C, \quad q_{34}=\beta S_{u 2}+\beta_{1}(1-\epsilon) S_{a 2}
\end{aligned}
$$

Hence $\dot{V}_{2}$ is negative definite and then the solution starting from any initial point satisfy the conditions (14a)-(14h), will approaches asymptotically to COVID-19 equilibrium point. Hence the proof is complete.

\section{Numerical Simulation}

In this section, we present the numerical simulation results and effect the parameters of the analytic results of system (1). Using MATLAB vergin 15 and $\mathrm{C}++$ software, we begin the simulation with a set of hypothetical values of parameters which are described in section 2, that we written in following

$$
\begin{gathered}
\Psi=3 ; \alpha=4 ; \beta_{1}=0.01 ; \sigma=0.3 ; \mu=0.1 ; \beta=0.03 ; \sigma_{1}=0.1 \\
\gamma=0.1 ; r=2 ; \epsilon=0.01, \theta=0.02 ; k=0.5 ; \mathcal{R}_{\circ}=818.1
\end{gathered}
$$

To know the impact the variation of awareness rate $\alpha$ on behavior of system (3), with keeping other parameters fixed as given in equation (15). We obtain the trajectories of system (3) to COVID-19 equilibrium point when the value of $\alpha=0,4$, which is shown in Fig. 2. 

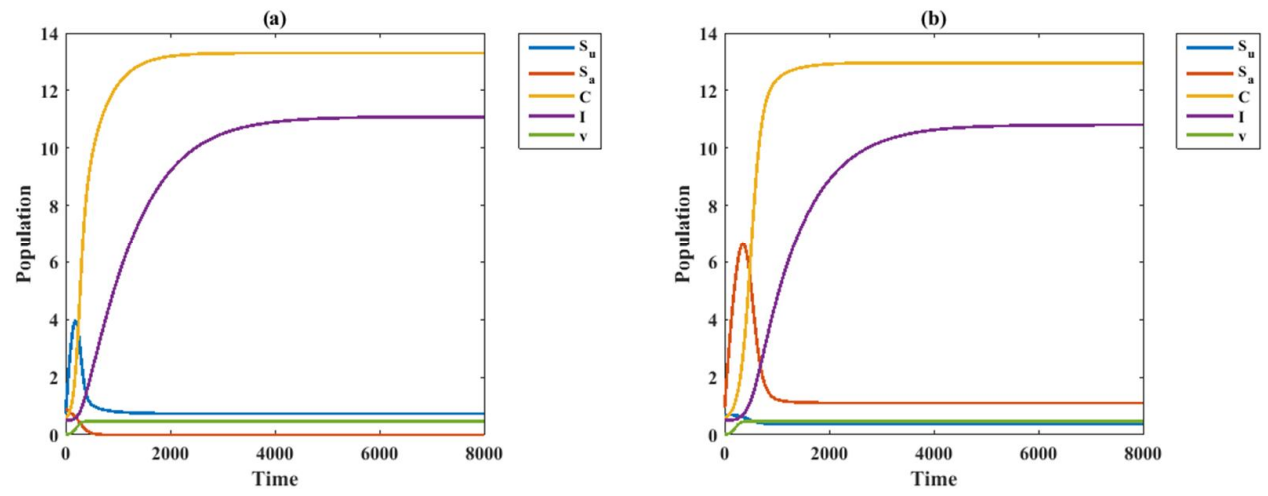

Fig. 2 Time Series plot for varying the awareness rate. (a) without awareness $\alpha=0$, (b) with awareness $\alpha=4$. With initial condition $(0.7,0.9,0.6,0.5,0.01)$.

We summarize that the increase the awareness rate just isn't enough to reduce the epidemic. And we observe that the number of $S_{a}$ are increasing. But the numbers of $S_{u}, C$ and $I$ are decreasing.

Furthermore, the effect of prevention rate of disease $\epsilon$ is not most different on effect the awareness rate if we change value it only. with keeping other parameters fixed as given in equation (15), which is shown that in Fig. 3.
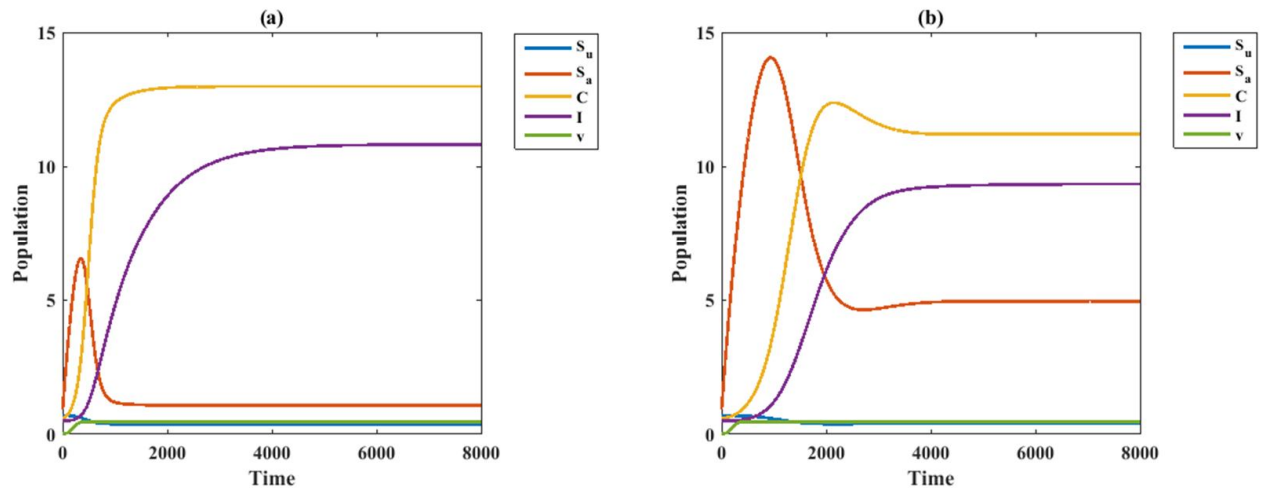

Fig. 3 Time Series plot for varying the prevention rate of disease. (a) without prevention of disease $\epsilon=0$, (b) with prevention of disease $\epsilon=0.8$. With initial condition $(0.7,0.9,0.6,0.5,0.01)$.

Clearly, the prevention rate of disease increase, we observe that the number of $S_{u}$ and $S_{a}$ are increasing. But the numbers of $C$ and $I$ are decreasing.

Now, we discuss effect the awareness rate and prevention rate of disease together, with reduce values of the contact rate on dynamical behavior of system (3). Hence, we can change in equation (15), became

$$
\begin{gathered}
\Psi=3 ; \alpha=4 ; \beta=\beta_{1}=0 ; \mu=0.1 ; \beta=0.03 ; \sigma=\sigma_{1}=0.01 \\
\gamma=0.1 ; r=2 ; \epsilon=0.8, \theta=0.02 ; k=0.5 ; \mathcal{R}_{\circ}=27.2
\end{gathered}
$$

We obtain the trajectories of system (3) to carrier free equilibrium, which is shown in Fig. 4. 

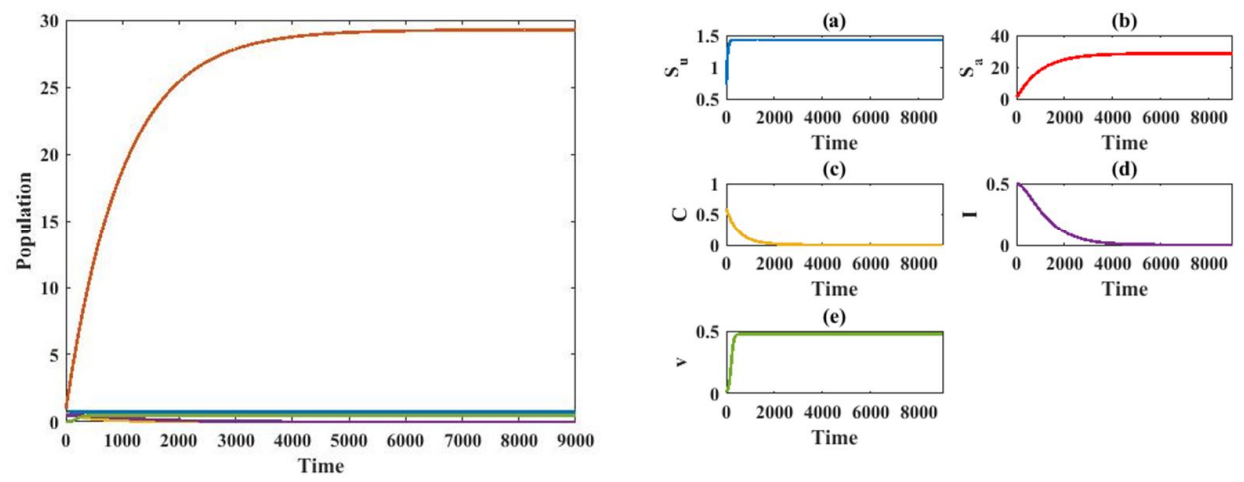

Fig. 4 Time Series of trajectories of system (3) to carrier disease point. (a) unaware susceptible, (b) aware susceptible, (c) carrier, (d) infected, (e) coronavirus. With initial condition $(0.7,0.9,0.6,0.5,0.01)$.

We summarize that, if the value of the awareness and prevention of disease rates increasing with reduce the contact rates. Then, we get the number of carrier and infected goes to zero and the trajectories of system (3) to $E_{1}=(1.4,28.5,0,0,0.47)$.

\section{Discussions and conclusions}

In this manuscript, an COVID-19 epidemic model is proposed and discussed. This model has three feasible equilibrium points are COVID-19 free, Carrier free and COVID-19. The results of the proposed work may be used to shown and understand the effect of awareness rate, prevention rate of disease and contact rate with carrier. The stability (local and global) of the model has been analyzed at all equilibrium points. it has been obtained that COVID-19 free is locally asymptotically stable if the condition (9) is holds and it is global stable if the conditions (12) is holds. While, the Carrier free point is locally asymptotically stable if the conditions (10a)-(10b) are hold and if conditions (13a)-(13c) satisfied we get it is globally asymptotically stable. Similarly, for COVID-19 point it has been obtained under certain conditions for locally as well as globally asymptotically stable. To validate the analytical results, we have executed the numerical simulations for investigating the dynamics of COVID-19 model. it has been obtained that the awareness factor only isn't enough to reduce the epidemic. Similarly result by prevention rate of disease. But we can reduce the epidemic dangerous by applied the all it together.

\section{Acknowledgements}

This work is supported by the National Natural Science Foundation of China (No. 11701495), Scientific and Technological Key Projects of Henan Province (No. 192102310193) and Nanhu Scholars Program for Young Scholars of XYNU. The authors thankful to acknowledge the reviewers for their valuable suggestions and comments and prof. Chin-Hong Park, Sung-Yell Song Editor in Chief of journal of Applied Mathematical and Computing. Their useful comments have contributed to the improvement of the authors work.

\section{References}

[1] J.D. Murray, 1989, Mathematical Biology, Biomathematics 19, Springer-Verlag, Berlin-Heidelberg-New York. 
[2] J. Hays, 2005, Epidemics and Pandemics: Their Impacts on Human History, ABC-CLIO. p. 193. ISBN: 1-85109-658-2.

[3] D. Barua, W. B. Greenough, 1992, Cholera, Springer, ISBN: 978-0-306-44077-9.

[4] C. C. Tan, 2006, SARS in Singapore key lessons from an epidemic. Ann Acad Med Singapore, 35, 345-349.

[5] T. Tsang and TH. Lam, 2003, SARS public health measures in Hong Kong. Respirology, 8, 46-48.

[6] A. Ahmed, R. Krumkamp and R. Reintjes, 2009, Controlling SARS a review on China response compared with other SARS-affecred countries. Trop Med Int Health, 14, 36-45.

[7] S. Riley, C. Fraser, CA. Donnelly, et al. 2003, Transmission dynamics of the etiological agent of SARS in Hong Kong impact of public health interventions. Science, 300, 1961-1966.

[8] World Health Organization. Coronavirus. World Health Organization, cited January 19, 2020. Available: https://www.who.int/health-topics/coronavirus.

[9] P. Zhou, XL. Yang, XG. Wang, B. Hu, L. Zhang, W. Zhang, et al., 2020, A pneumonia outbreak associated with a new coronavirus of probable bat origin. Nature. https://doi.org/10.1038/s41586-020-2012-7.

[10] N. Zhu, D. Zhang, W. Wang, X. Li, B. Yang, J. Song, et al., 2020, A novel coronavirus from patients with pneumonia in China, 2019. N. Eng. J. Med. https://doi.org/10.1056/NEJMoa2001017.

[11] World Health Organization. Novel Coronavirus - Japan (ex-China). World Health Organization. cited January 20, 2020. Available:

https://www.who.int/csr/don/17-january-2020-novel-coronavirus-japan-exchina/en/.

[12] Q. Li, X. Guan, P. Wu, et al., 2020, Early transmission dynamics in Wuhan, China, of novel coronavirus-infected pneumonia. N. Eng. J. Med., published online Jan 29. https://DOI:10.1056/NEJMoa2001316.

[13] Horn R.A. and Johnson C.R. 1985. matrix analysis. Cambridge University press. 
Figures

\section{The Effect of Carrier to outbreak the COVID-19}

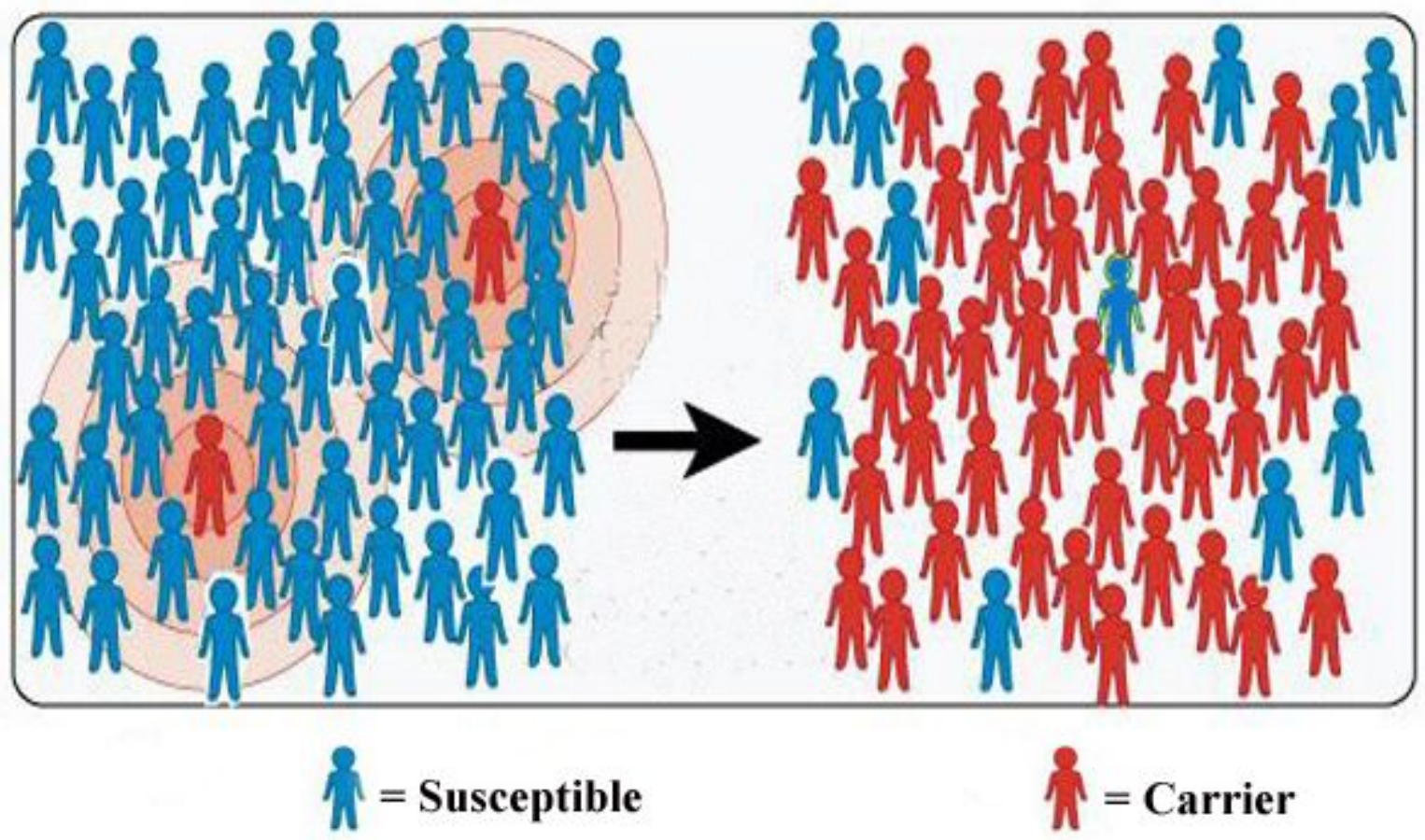

Figure 1

Outbreak Corona-virus due to Carrier by contact
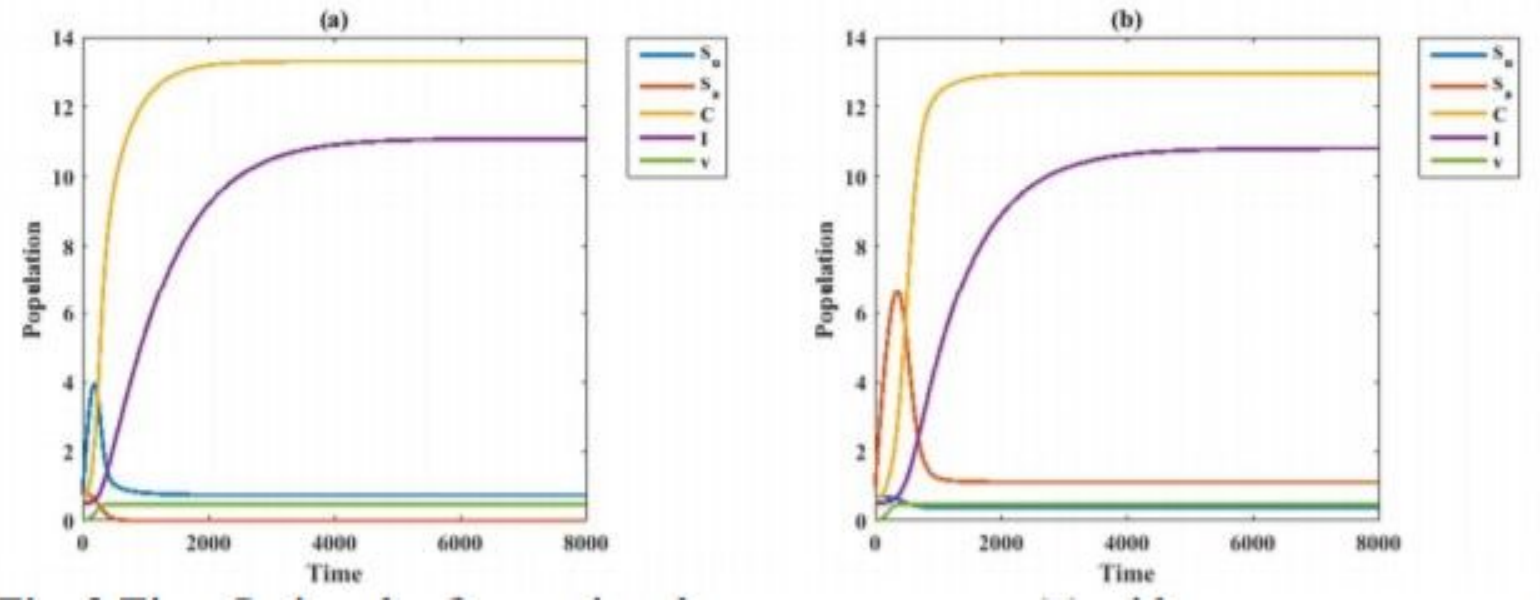

Figure 2 
Time Series plot for varying the awareness rate. (a) without awareness $a=0$, (b) with awareness $a=4$. With initial condition $(0.7,0.9,0.6,0.5,0.01)$.
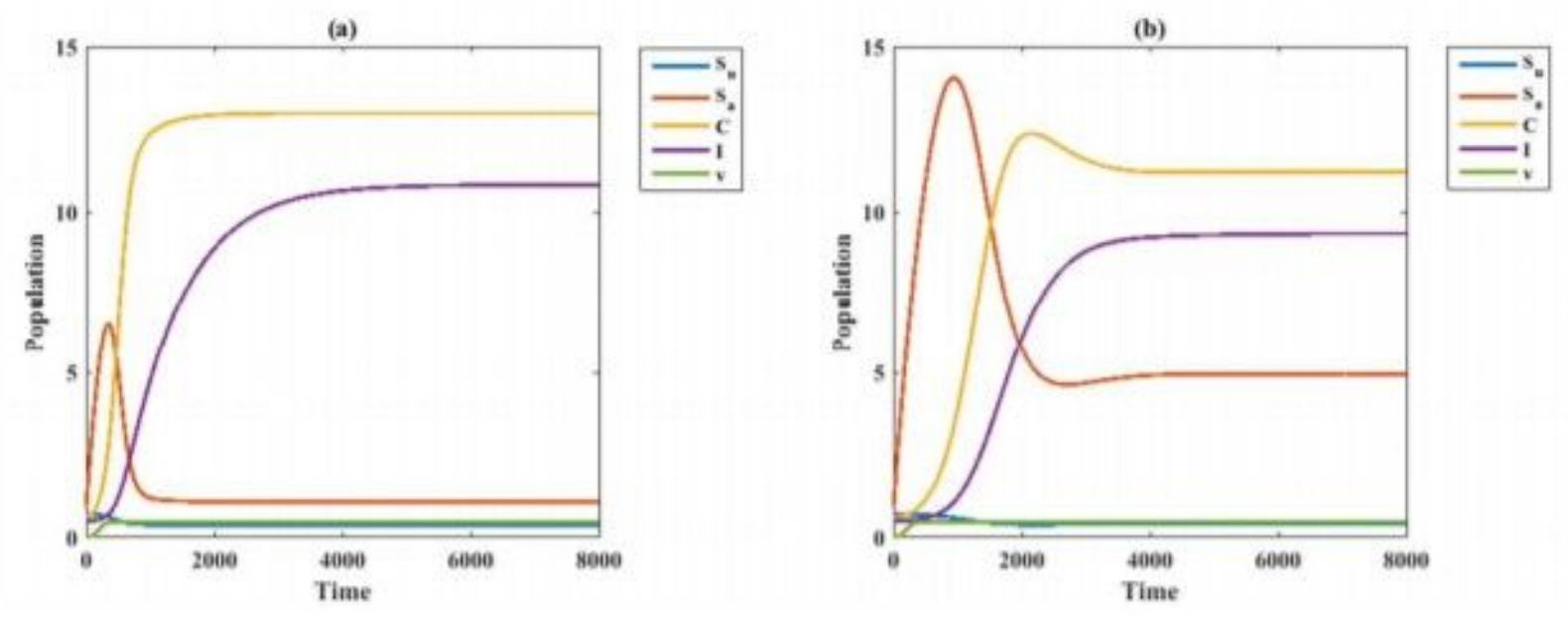

\section{Figure 3}

Time Series plot for varying the prevention rate of disease. (a) without prevention of disease $\square e=0$, (b) with prevention of disease $\mathrm{De}=0.8$. With initial condition $(0.7,0.9,0.6,0.5,0.01)$.

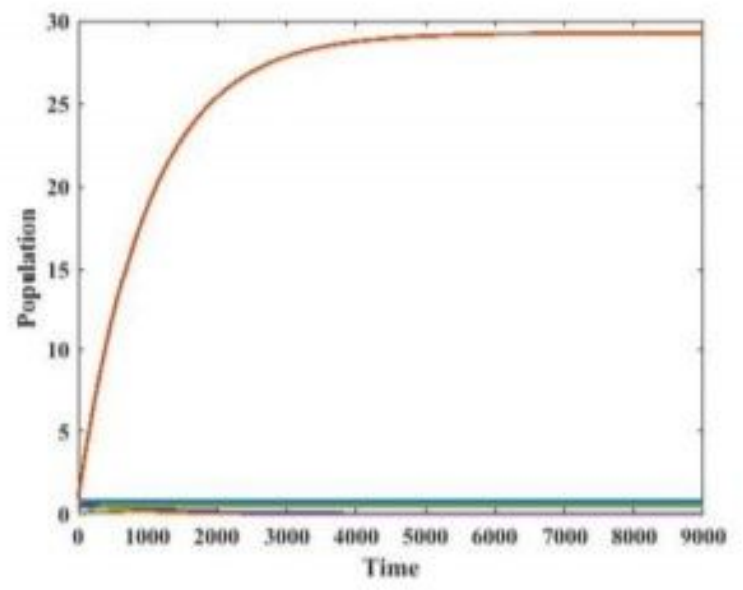

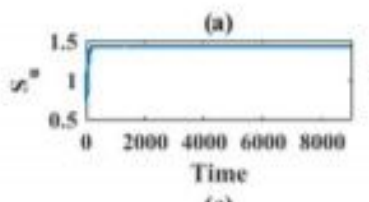

(c)

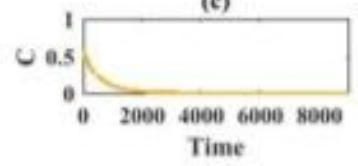

(e)

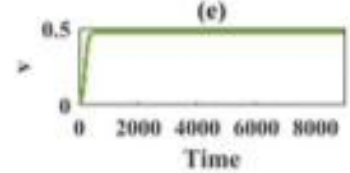

(b)

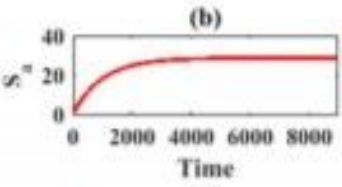

(d)

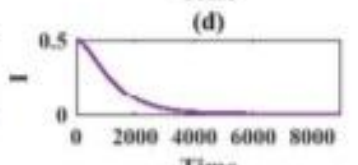

Time

\section{Figure 4}

Time Series of trajectories of system (3) to carrier disease point. (a) unaware susceptible, (b) aware susceptible, (c) carrier, (d) infected, (e) coronavirus. With initial condition $(0.7,0.9,0.6,0.5,0.01)$. 